\title{
Metodología para la Evaluación de la Calidad de Estrategias Didácticas de Cursos a Distancia (MACCAD)
}

\author{
Myriam G. Llarena \\ Universidad Nacional de San Juan, Facultad de Ciencias Exactas, Físicas y Naturales, \\ Av. Ignacio de la Roza y Meglioli, 5423 Rivadavia, San Juan-Argentina \\ (e-mail: mgllarena2000@yahoo.com.ar)
}

\section{Resumen}

Se describe un modelo para evaluar la calidad de aspectos didáctico-pedagógicos de cursos no presenciales. Se realizó en primera instancia un análisis teórico del modelo y luego un diseño exploratorio-descriptivo. Como resultado del análisis teórico se determinó un modelo que propone los criterios utilizados en los instrumentos generados para evaluar la calidad de la interactividad e interacción en la modalidad de enseñanza a distancia. Se muestra que esta metodología permite además identificar los criterios más significativos para los alumnos en la determinación de su satisfacción general respecto del curso, las fortalezas y debilidades del mismo y la fiabilidad del instrumento de evaluación utilizado.

\section{Methodology for Evaluating the Quality of Teaching Strategies Course Distance (MACCAD)}

\begin{abstract}
A model for evaluating the quality of teaching aspects in non-presencial courses is described. A theoretical analysis is first done to then perform a descriptive and exploratory design. As a result of the theoretical analysis, a model that proposes the criteria used in the instruments generated to evaluate the quality of interaction and interactivity in the distance mode teaching. Its is shown that the methodology also allows to identify the most important criteria for students to determine their overall satisfaction with respect to the course, the strengths and weaknesses of it and the reliability of the assessment tool used.
\end{abstract}

Keywords: distance education, evaluation, quality indicators, teaching strategies 


\section{INTRODUCCIÓN}

La educación es un perfeccionamiento intencional, que cada hombre realiza en busca de su desarrollo integral. La necesidad de un aprendizaje continuo, a lo largo de toda la vida, resulta una condición para la inserción en el mundo globalizado actual. La educación a distancia, surge como una alternativa de educación que otorga al individuo un alto grado de autonomía, permitiéndole compatibilizar las exigencias de capacitación y perfeccionamiento, con las limitaciones espaciotemporales impuestas por sus obligaciones laborales.

Litwin (2000), refiere a las especificidades de la modalidad de educación no presencial, que se pueden resumir en: mediatización de las relaciones entre docentes y alumnos, acento en la autonomía de los estudiantes para elegir espacio y tiempo para el estudio, aprendizaje colaborativo, importancia del material didáctico que favorece la autogestión del aprendizaje y utilización de multiplicidad de recursos pedagógicos para favorecer la construcción de los conocimientos. Garcia Aretio (2002), señala que el diálogo o comunicación didáctica entre el que enseña y el que aprende es el elemento central en el proceso de E-A para esta modalidad y que este diálogo se establece a través de medios preproducidos y por vías de comunicación.

La Facultad de Ciencias Exactas, Físicas y Naturales de la Universidad Nacional de San Juan, no ajena a las demandas de la sociedad, está realizando propuestas de cursos bajo la modalidad no presencial y es su preocupación cristalizarlas en un marco de calidad. La conveniencia de medir y evaluar la calidad de sus propuestas educativas ya no es tema de debate, está instalado en el ámbito de las universidades; el motivo de análisis está centrado ahora en determinar aspectos, características e instrumentos de evaluación de las actividades del proceso educativo que deben ser evaluados. Rubio (2003), indica que las herramientas de evaluación que se utilizan en los contextos de formación virtual, se refieren principalmente a los aspectos de gestión organizativa, a la relación costo-beneficio, aspectos que si bien son importantes no son suficientes para un proceso en el que lo más significativo es la enseñanza/ aprendizaje. La modalidad a distancia requiere una manera particular de promover el aprendizaje, siendo su rasgo distintivo la mediatización de las relaciones entre docentes y alumnos- separados físicamente, en espacio, tiempo o ambos a la vez-, entre materiales y alumnos y de ellos entre sí. Por ello se decide acotar la tarea, a la evaluación de la interacción entre los distintos actores del proceso educativo e interactividad del alumno con el material de aprendizaje y la tecnología, por ser éstos, aspectos fundamentales en el proceso de enseñanza/aprendizaje bajo la modalidad a distancia. Para generar mecanismos que aseguren la calidad de estos procesos, surge MACCAD (Metodología para Autoevaluación de Calidad de Cursos a Distancia), que da respuesta a la siguiente problemática:

¿Qué instrumentos se podrán utilizar para evaluar la interacción e interactividad, fundamentales en el proceso de enseñanza aprendizaje de los cursos no presenciales?

¿Qué indicadores deberán considerarse?

¿Cómo se puede determinar la fiabilidad de los instrumentos utilizados?

MACCAD propone tres etapas, en la primera y a partir de una investigación teórica se determina por qué evaluar - Objetivos de Evaluación -, qué evaluar - Modelo de Evaluación - y cómo y con qué evaluar - Proceso de Evaluación -. Como resultado de esta etapa se generan los cuestionarios de satisfacción utilizados como instrumentos de evaluación. Las otras dos etapas, destinadas a comprobar y mejorar la calidad, requieren de un diseño exploratorio-descriptivo. El análisis de los indicadores obtenidos, utilizando una metodología cuanti/cualitativa, permite determinar la calidad de los procesos evaluados y realizar propuestas de mejoras. Finalmente especificar la fiabilidad de los instrumentos permite tener confianza de que ellos reflejan realmente lo que los usuarios quieren expresar.

\section{METODOLOGIA MACCAD}

Una metodología de evaluación debe estar sustentada en una concepción de calidad. Para algunos la calidad está centrada en el servicio o producto en sí: "adecuado para el uso" (Juran y Gryman, 1995), para otros en cambio está centrada en el usuario: "conformidad con los requerimientos" (Crosby, 1998). La Calidad Total requiere de ambos requisitos, calidad del servicio 
y satisfacción del usuario. MACCAD está sustentada en esta filosofía de calidad, considera no sólo la calidad del proceso sino también la satisfacción del alumno; conocer lo que él percibe permitirá a la institución que ofrece el servicio, tomar decisiones tendientes a satisfacer sus necesidades y exigencias. Se adaptó la metodología basada en el modelo de desarrollo y utilización de cuestionarios de satisfacción de clientes, propuesta por Hayes (1992). Este autor, sugiere el uso de cuestionarios de satisfacción, formados por frases llamadas artículos de satisfacción, que conforman los distintos criterios o dimensiones a evaluar. Esta decisión se justifica en que en dicha metodología se especifica claramente como generar y evaluar científicamente los instrumentos de evaluación.

A continuación se describe como se concretó la primera etapa propuesta por MACCAD, Planificación de la evaluación, en la que se gestiona la calidad. Esta etapa comienza con la determinación de los objetivos de evaluación y consiste en una investigación teórica. Se analizaron informes de investigaciones efectuadas en universidades e instituciones de punta en esta temática, considerando las Guías de Buenas Prácticas que ellas pudieran haber generado. Como resultado de este análisis se determinó el Modelo de Evaluación, que permite descomponer el concepto de calidad en forma jerárquica en subconceptos más concretos de manera de facilitar su evaluación. Un modelo de evaluación debe establecer Criterios e Indicadores, que estén en relación con los objetivos establecidos por la institución que ofrece el curso. Criterios son aquellos factores considerados críticos para la calidad del curso- servicio-, tanto desde el punto de vista de los especialistas en educación de la institución que lo propone, como del de los alumnos usuarios- del mismo. Respecto al concepto de Indicador, referentes de la UNESCO puntualizan que un indicador es una señal o indicio que indica algo, que muestra qué sucede, son estadísticas que permiten establecer juicios de valor acerca de aspectos claves del sistema educativo. Si bien los criterios e indicadores deben adecuarse a las características del curso, la disciplina y el perfil de los alumnos, entre otros factores, se considera que los siguientes son fundamentales para la evaluación de la interacción e interactividad de los cursos a distancia.

\section{Criterios para evaluar interactividad}

Para analizar la interactividad alumno/ material se consideraron dos criterios Aspectos Didácticos y Aspectos Curriculares y para determinar la calidad de la interactividad alumno/ recursos tecnológicos, se consideró el criterio Entorno Tecnológico. El criterio Aspectos Didácticos se refiere a la funcionalidad y adecuación de los materiales presentados. Entendiendo por materiales a todos los recursos de aprendizaje que se ponen a disposición del alumno en la plataforma: contenidos, guías didácticas, actividades prácticas, software específico, etc. El criterio Aspectos Curriculares se refiere a la profundidad, validez, adecuación y cantidad de contenidos propuestos.

Para evaluar la interactividad del alumno con los recursos tecnológicos, se consideró el criterio Entorno Tecnológico que consta de 2 subcriterios: accesibilidad, que contempla la distribución y facilidad de acceso de los distintos materiales elaborados por el equipo responsable del curso contenidos, guías didácticas, actividades prácticas, documentos de apoyo- y la usabilidad de los recursos provistos por la plataforma- correo interno, foro, chat-.

\section{Criterios para evaluar interacción}

La interacción entre los distintos actores, se refiere a la interacción alumno/tutor y alumnos entre sí. Para ello se consideró el criterio Tutorías en el que se evalúa el profesionalismo, responsabilidad y compromiso del tutor en el cumplimiento de sus tareas, como así también las características, tipo de consultas y herramientas más utilizadas. Por la importancia que en este proceso educativo tienen los aspectos referidos al aprendizaje colaborativo, la interacción alumno/alumno se evalúa a través de la experiencia vivenciada por ellos en las actividades grupales y vía foro. Se ha considerado también el criterio Autogestión del Aprendizaje, para evaluar la adecuación y validez de las propuestas para favorecer la formación del alumno: actividades y tiempos planificados entre otros. La metodología de Hayes (1992) determina agregar el criterio Satisfacción General, formado por artículos menos específicos, para evaluar la calidad general del curso También son incluidas en los cuestionarios preguntas abiertas destinadas a conocer los aspectos que fueron vivenciados como positivos y/o negativos por los alumnos, para la identificación de fortalezas y debilidades del curso. 


\section{Indicadores}

Los indicadores utilizados para determinar la calidad de los procesos de interacción e interactividad, basada en la satisfacción de los alumnos, para variables cuantitativas son la media aritmética, la dispersión y el coeficiente de correlación. La media aritmética es el promedio de todas las puntuaciones asignadas, permite determinar la tendencia central de las puntuaciones obtenidas, tanto para los criterios como para los artículos de satisfacción que los constituyen. La dispersión, indica la variabilidad o diseminación de dichos valores respecto de ese valor medio. Representa el "grado de unanimidad" de la percepción de calidad. Las siguientes son las expresiones matemáticas que permiten calcular estos indicadores:

Media Aritmética

$$
\bar{X}=\sum_{i=1}^{i=n} x i / \mathrm{n} \quad \bar{X}=\left(\mathrm{x}_{1}+\mathrm{x}_{2}+\ldots+\mathrm{x}_{\mathrm{n}}\right) /
$$

$\mathrm{n}$

Donde xi corresponde a la puntuación asignada por el alumno i-ésimo y $\mathrm{n}$ es la cantidad de alumnos que contestaron el cuestionario.

Dispersión

$$
\sigma=\sqrt{\frac{\sum_{i=1}^{n}\left(x_{i}-\bar{X}\right)^{2}}{n-1}}
$$

La dispersión es un promedio de los desvíos de cada puntuación obtenida, respecto de la media de todas las puntuaciones. Estos indicadores se utilizan para el caso de variables cuantitativas y se evalúan en una escala comprendida entre 1 (satisfacción mínima) y 4 puntos (máximo nivel de satisfacción). Para la interpretación de la dispersión se apela también a los gráficos de frecuencia. Para las variables cualitativas se han calculado frecuencias y porcentajes. El Indicador Coeficiente de Correlación, permite conocer los criterios de calidad que están más relacionados con la satisfacción general de los alumnos. El resultado de este coeficiente es un número comprendido entre -1 y 1 . El valor 1 indica una relación lineal perfecta y positiva entre las variables, -1 representa también una relación perfecta pero negativa, mientras que el valor 0 señala que no existe relación entre ellas. En general los valores que se obtendrán no son exactamente los tres mencionados, pero si el valor obtenido es próximo a 1, indica relación fuerte entre las variables estudiadas.

La tabla 1 muestra algunos criterios del cuestionario de satisfacción confeccionado para evaluar la calidad de las estrategias didáctico/pedagógicas del curso Gravimetría y sus Aplicaciones Tectónicas. Este curso con modalidad a distancia, destinado a Geofísicos, Geólogos y profesionales relacionados con las Ciencias de la Tierra, consistió de dos módulos. Se planificaron dos evaluaciones, basadas en la satisfacción de los alumnos, una al finalizar cada uno los módulos. La evaluación planificada para el final del primer módulo -orientada a la mejorapermitiría realizar las modificaciones necesarias antes de comenzar el segundo. La evaluación planificada para el final del segundo módulo, con carácter de control, permitiría determinar objetivos alcanzados, aspectos positivos a ser potenciados y aspectos negativos a corregir para próximas propuestas.

Los artículos considerados fueron consensuados entre los integrantes del área pedagógica y los docentes del área disciplinar. Se incluyó el criterio Satisfacción General, como sugiere la metodología de Hayes (1992). 
Tabla 1: Instrumento de evaluación

ENCUESTA DE CALIDAD

Hemos concluido nuestra experiencia de aprendizaje a distancia. Este instrumento de evaluación nos permitirá determinar si sus expectativas fueron satisfechas. Marque con una cruz la opción elegida.

NOTA: Si la opción seleccionada es Generalmente(3), A veces(2) o Nunca(1), por favor especifique las dificultades o disconformidades. Su opinión representa un valioso aporte, ya que ella nos permitirá determinar debilidades y fortalezas de esta experiencia. Su sinceridad será muy apreciada. Gracias.

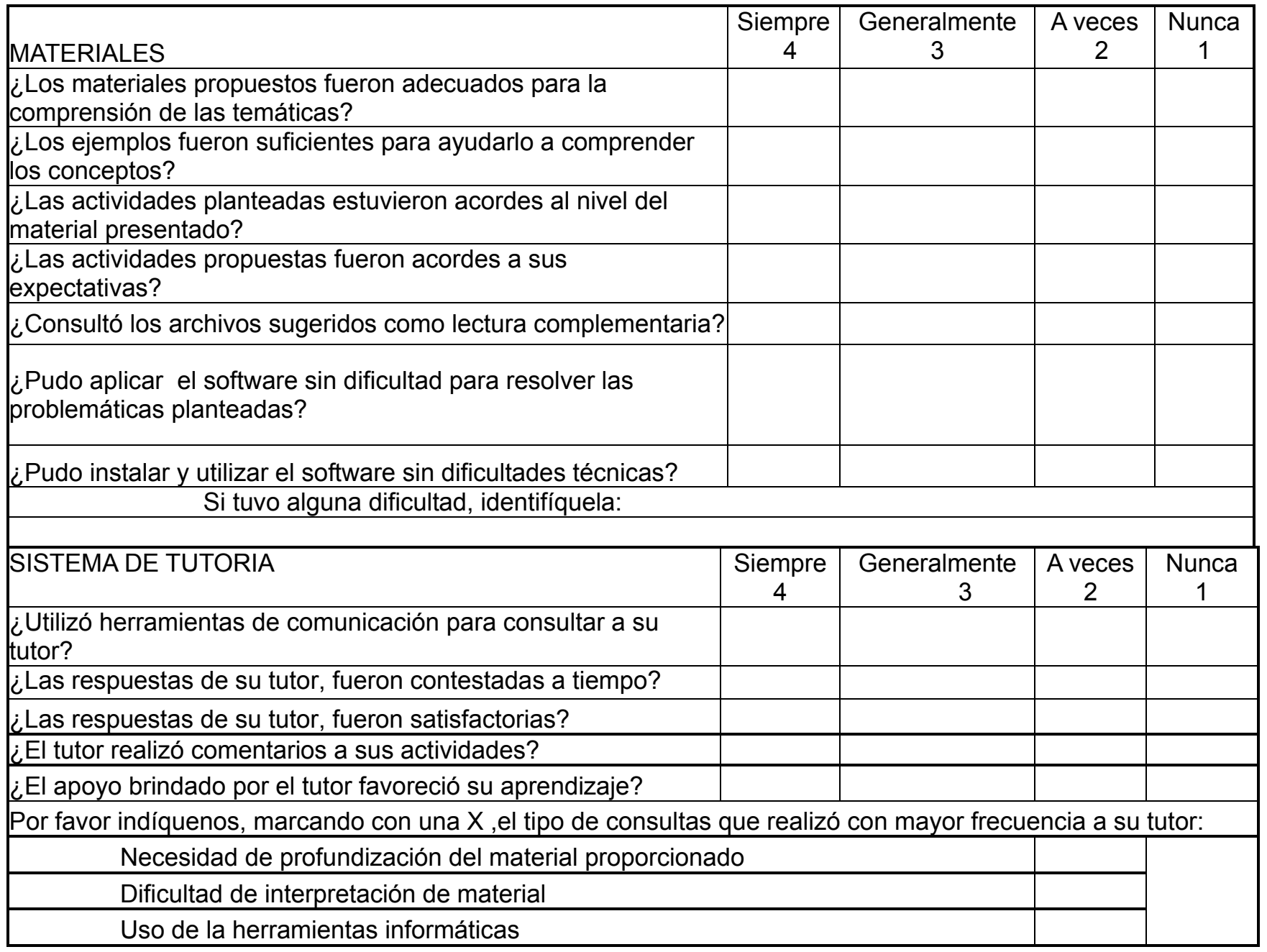

\begin{tabular}{|c|c|c|c|c|c|}
\hline ENTORNO TECNOLOGICO & $\begin{array}{c}\text { Siempre } \\
4\end{array}$ & \multicolumn{2}{|c|}{$\begin{array}{c}\text { Generalmente } \\
3 \\
\end{array}$} & $\begin{array}{l}\text { A veces } \\
2 \\
\end{array}$ & $\begin{array}{c}\text { Nunca } \\
1\end{array}$ \\
\hline \multicolumn{6}{|l|}{ Accesibilidad } \\
\hline \multicolumn{6}{|l|}{$\begin{array}{l}\text { ¿La distribución de los materiales en el entorno fue } \\
\text { adecuada a sus necesidades? }\end{array}$} \\
\hline \multicolumn{6}{|l|}{ ¿Accedió sin dificultades a los servicios? } \\
\hline \multicolumn{6}{|l|}{ Usabilidad } \\
\hline \multicolumn{6}{|l|}{ ¿Pudo manejar los recursos de la plataforma sin dificultad? } \\
\hline \multicolumn{6}{|l|}{$\begin{array}{l}\text { ¿Los recursos ofrecidos por la plataforma se adecuaron a } \\
\text { sus necesidades? }\end{array}$} \\
\hline \multicolumn{6}{|l|}{ Identifique sus necesidades insatisfechas } \\
\hline SATISFACCIÓN GENERAL & $\begin{array}{c}\text { Muy buena } \\
4\end{array}$ & $\begin{array}{c}\text { Buena } \\
3\end{array}$ & $\begin{array}{l}\text { Regular } \\
2\end{array}$ & & ala \\
\hline \multicolumn{6}{|l|}{ Realice el balance de su experiencia respecto de: } \\
\hline \multicolumn{6}{|l|}{ Materiales de aprendizaje } \\
\hline \multicolumn{6}{|l|}{ Sistema de tutoría } \\
\hline Recursos de la plataforma & & & & & \\
\hline
\end{tabular}




\section{RESULTADOS Y DISCUSIÓN}

Los siguientes son los resultados de los indicadores correspondientes al curso de posgrado "Gravimetría y sus Aplicaciones Tectónicas":

\section{Indicadores Media y dispersión}

La tabla 2 muestra los indicadores, media y dispersión obtenidos para los criterios evaluados, al procesar los cuestionarios de satisfacción de cada uno de los módulos. Los valores medios obtenidos para los criterios considerados, reflejan en general un alto grado de conformidad y las pequeñas dispersiones indican unanimidad en las opiniones en ambos módulos. La figura 1, que ofrece una visión general respecto del grado de satisfacción de los alumnos, indica que el promedio de satisfacción ha aumentado en los distintos criterios para el segundo módulo. Esto señala que las acciones llevadas a cabo al finalizar el primer módulo, tendientes a mejorar la propuesta del segundo, han sido pertinentes. La tabla 2 resulta de gran utilidad al evaluador, ya que le permite una percepción rápida de la situación y centrar especial atención en aquellos criterios en que los alumnos manifiestan mayor disconformidad. En este caso para ambos módulos, el criterio en el que los alumnos presentan dificultad es Materiales. El análisis de los indicadores de los distintos artículos de este criterio permitirá dilucidar la causa de la disconformidad. La tabla 3 muestra un comparativo de los indicadores promedio y dispersión correspondientes a algunos artículos del criterio Materiales.

Tabla 2: Comparativo Indicadores Criterios Módulos I y II

Módulo I
\begin{tabular}{|c|c|c|}
\hline CRITERIOS & Media & Dispersión \\
\hline SISTEMA TUTORIAS & 3,79 & 0,07 \\
\hline TECNOLOGÍA & 3,59 & 0,13 \\
\hline MATERIALES & 3,23 & 0,27 \\
\hline
\end{tabular}

\begin{tabular}{|c|c|c|} 
Módulo II \\
\begin{tabular}{|c|c|c|}
\hline CRITERIOS & Media & Dispersión \\
\hline SISTEMA TUTORIAS & 3,86 & 0,18 \\
\hline TECNOLOGÍA & 3,98 & 0,03 \\
\hline MATERIALES & 3,61 & 0,59 \\
\hline
\end{tabular}
\end{tabular}

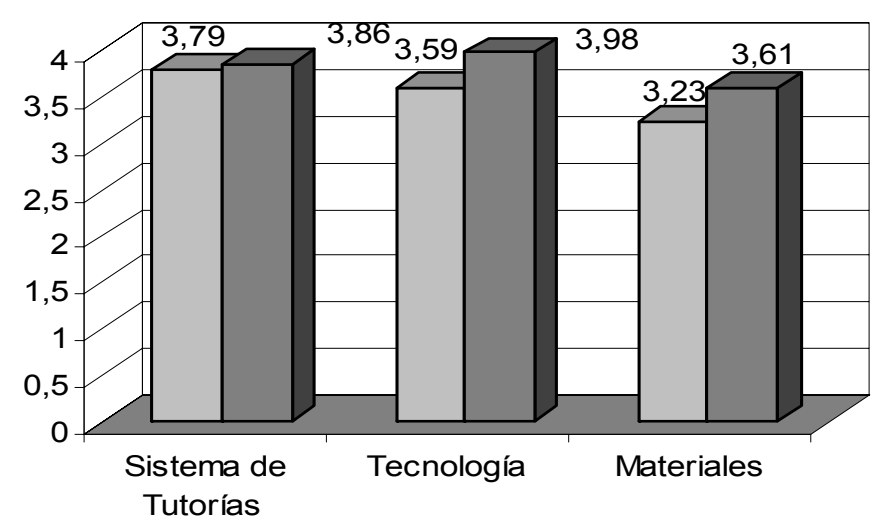

Fig. 1: Comparativo Promedios Módulos I ( $\square$ ) y II ( $\square$ )

Los valores que se observan el la tabla 3 indican que los alumnos en el segundo módulo se habituaron al uso de la plataforma, todos asignaron al artículo ¿La distribución de los materiales en el entorno fue adecuada?, el máximo puntaje. Respecto de los reajustes realizados para lograr una mejor comprensión en las temáticas tratadas, atendiendo a las sugerencias realizadas en la primera encuesta, evidentemente resultaron satisfactorios ya que el puntaje promedio se incrementó de 3,25 a 3,86. El último artículo se debe considerar con especial atención, para lo cual se apela a la opinión de los alumnos, teniendo en cuenta que en el cuestionario se solicita especificar la causa de la disconformidad en caso de asignar puntajes inferiores a 3 puntos. Esto indican algunos alumnos:

"Por desconocimiento de DOS tuve problemas para la carga de datos (espacios, tabulaciones), 
tengo entendido que el DOS no es del todo compatible con el Windows 2000.."

"En mi caso el problema consistió en el desconocimiento de DOS, en lo que asumo mi responsabilidad, una solución sería trabajar con programas más amigables.

Tabla 3: Indicadores Artículos Criterio MATERIALES

\begin{tabular}{|l|c|c|c|c|}
\cline { 2 - 5 } \multicolumn{1}{c|}{} & \multicolumn{2}{c|}{ Módulo I } & \multicolumn{2}{c|}{ Módulo II } \\
\cline { 2 - 5 } \multicolumn{1}{c|}{} & Media & Dispersión & Media & Dispersión \\
\hline MATERIALES & 3,23 & 0,37 & 3,61 & 0,59 \\
\hline ¿La distribución de materiales en el entorno fue adecuada ? & 3,63 & 0,52 & 4 & 0 \\
\hline ¿Fueron suficientes para la comprensión de las temáticas? & 3,25 & 0,46 & 3,86 & 0,38 \\
\hline ¿Pudo utilizar los software sin dificultad?- Aspecto didáctico- & 3,38 & 0,92 & 3,14 & 0,90 \\
\hline ¿Tuvo dificultades técnicas con el software? & 2,63 & 1,06 & 2,67 & 0,84 \\
\hline
\end{tabular}

Así como los indicadores media y dispersión se utilizan para determinar las debilidades de la propuesta, también permiten visualizar sus fortalezas. Este es el caso de dos de los artículos correspondientes del criterio Sistema de Tutorías, que se muestran en la tabla 4.

Tabla 4: Indicadores de dos artículos correspondientes al Criterio SISTEMA TUTORIAS

\begin{tabular}{|l|c|c|}
\cline { 2 - 3 } \multicolumn{1}{c|}{} & Media & Dispersión \\
\hline ¿Las respuestas de su tutor, fueron satisfactorias? & 4 & 0 \\
\hline ¿El apoyo brindado por el tutor favoreció sus aprendizajes? & 4 & 0 \\
\hline
\end{tabular}

Para estos docentes disciplinares, que tuvieron su primera experiencia en esta modalidad de enseñanza, es muy gratificante que todos los alumnos hayan evaluado con el máximo puntaje el apoyo brindado en el proceso de acompañar sus aprendizajes.

En el cuestionario también se utilizaron variables cualitativas, tal es el caso de la evaluación del tipo de consulta realizada con mayor frecuencia al tutor. Los resultados obtenidos se muestran en la tabla 5.

Tabla 5: Tipo de consulta que realizó con mayor frecuencia al tutor

\begin{tabular}{|c|c|c|}
\hline & Frecuencia & Porcentaje \\
\hline Necesidad de profundización del material & 3 & $43 \%$ \\
\hline Dificultad de interpretación de material & 3 & $43 \%$ \\
\hline Uso de la herramientas informáticas & 5 & $71 \%$ \\
\hline
\end{tabular}

Estos resultados ponen nuevamente de manifiesto que los alumnos tuvieron dificultad en el uso del software. Como puede observarse, la suma de los porcentajes no es $100 \%$ debido a que es una consulta de opciones múltiples.

\section{Indicador Coeficiente de Correlación}

Este coeficiente permite identificar los Criterios más importantes para los alumnos a la hora de evaluar su satisfacción respecto del curso en general. Los resultados obtenidos para ambos módulos se muestran en la tabla 6. 
Tabla 6: Comparativo Coeficiente Correlación Módulos I y II

\begin{tabular}{|l|c|c|}
\cline { 2 - 3 } \multicolumn{1}{c|}{} & MODULO I & MODULO II \\
\hline Criterios relacionados & Valor Correlación & Valor Correlación \\
\hline Correlación Tutorías/ Satisf. Gral & 0,85 & 0,16 \\
\hline Correl. Tecnología / Satisf. Gral & 0,50 & 0,56 \\
\hline
\end{tabular}

¿Qué indican estos coeficientes? Si bien desde el punto de quienes realizan la propuesta del curso todos los criterios considerados son importantes, para este grupo de alumnos en el caso del primer módulo, el criterio más significativo en la determinación de su satisfacción general del curso es el Sistema de Tutorías. Esto es comprensible ya que el rol de tutor o docente que guía y acompaña adquiere un papel fundamental cuando el proceso es totalmente a distancia y teniendo en cuenta además que para la mayoría de los alumnos era su primera experiencia en esta modalidad de enseñanza/aprendizaje. En el módulo II, se visualiza que ya no es tan importante la necesidad de apoyo por parte del tutor, debido a que a lo largo del curso el alumno ha vivenciado instancias de aprendizaje con sus pares. En este caso el criterio más significativo, aunque con una relación no muy fuerte, es la tecnología.

\section{Determinación de Fortalezas y Debilidades}

Otra de las potencialidades de la metodología es la de permitir identificar fortalezas y debilidades de la propuesta. Conocer los aspectos señalados como negativos por los alumnos, ayuda a reconocer las debilidades. Incluir además los gráficos de frecuencia, permite determinar si las opiniones corresponden a casos puntuales o es un juicio generalizado. En este último caso es conveniente conocer si la respuesta corresponde a grupos con determinadas características, para establecer acciones correctivas pertinentes. Para el curso considerado, entre los aspectos negativos señalados, se destacan los problemas técnicos del software utilizado, tanto en su instalación como para el tratamiento de los datos. No son casos aislados, por lo que deberán ser considerados para próximos cursos. Los aspectos señalados como positivos, son las fortalezas, las que deberán ser consideradas por los organizadores del curso para potenciarlos en futuras propuestas y determinar si ellos han sido el resultado de acciones concretas. Un aspecto positivo a destacar en este curso, es haber logrado la concientización de los docentes disciplinares, que en un principio de mostraron reticentes acerca de la necesidad de incorporar instancias de interacción entre alumnos en el proceso de enseñanza aprendizaje. Así lo expresa un tutor: "En los investigadores de este país- posiblemente por razones de fechas-,no observé el mismo interés que noté en los investigadores de otros países que se mostraron entusiasmados con el tema. Sería conveniente de plantearse otro curso de iguales características solicitar foros quizás desde la unidad 3 o 4 ..."

\section{Criterios que requieren consideración especial}

La tabla 7 muestra los valores promedios de satisfacción para los distintos criterios, ordenados de mayor a menor nivel de satisfacción y los coeficientes de correlación de los distintos criterios, respecto de la satisfacción general, también ordenados descendentemente.

Tabla 7: Promedio Criterios y Correlación Criterios/Satisfacción General

\begin{tabular}{|l|c|l|c|}
\hline Criterios & Media & Criterios relacionados & Correlación \\
\hline Recursos Plataforma & 3,98 & Recursos.Plataf / Satisf. Gral & 0,56 \\
\hline Tutoría & 3,86 & Autogestión/ Satisf. Gral & 0.55 \\
\hline Materiales de Aprendizaje & 3,61 & Tutoría/ Satisf. Gral & 0,16 \\
\hline Autogestión Aprendizaje & 3,29 & Materiales/ Satisf. Gral & $-0,034$ \\
\hline
\end{tabular}


Las variables puntaje promedio de cada criterio y coeficiente de correlación pueden relacionarse construyendo una tabla de doble entrada (Herrera et al., 2007). Teniendo en cuenta que el puntaje asignado varía de 1 a 4 puntos, se considera un puntaje satisfactorio aquel que supere 3.5 puntos y una correlación considerable la que tenga valor mayor o igual a 0.55 . La sistematización de la tabla 8 permite identificar aquellos criterios que siendo significativos en la determinación de la satisfacción general del alumno, obtuvieron un puntaje promedio bajo, por lo que merecen una consideración especial.

Tabla 8: Relación Promedio / Significación

\begin{tabular}{|c|l|c|}
\hline \hline Correlación & $>3.50$ & $<=3.50$ \\
\hline$>=0.55$ & Recursos Plataforma & Autogestión Aprendizaje \\
\hline$<0.55$ & Tutorías Materiales & \\
\hline
\end{tabular}

Para el curso evaluado los criterios que resultaron significativos son Recursos de la Plataforma y Autogestión del Aprendizaje, siendo este último el que habrá que considerar especialmente ya que el promedio obtenido es de 3,29. Es importante destacar que el criterio Autogestión del Aprendizaje, que resultó significativo en la evaluación de la satisfacción general del curso, fue incorporado en el segundo módulo a partir de sugerencias realizadas por los alumnos en la encuesta efectuada al finalizar el primer módulo. Del análisis de los puntajes asignados a los artículos que conforman dicho criterio, se observa menor satisfacción en lo que respecta a los tiempos planificados para la entrega de las actividades. Este aspecto deberá ser contemplado en futuras propuestas del curso.

\section{Acerca de la fiabilidad del instrumento}

Se entiende por fiabilidad al grado en que las mediciones están libres de la desviación producida por los errores casuales, esto es, el grado de coincidencia entre los valores obtenidos a partir de los cuestionarios y la verdadera opinión de los usuarios. Se han desarrollado diferentes enfoques y métodos para determinar la fiabilidad. Spearman (1930), realiza un propuesta pionera pero aún vigente, fundamentada en la Teoría Clásica de los Tests. De los tres métodos para medir la fiabilidad propuestos en el marco de esta teoría, el método de consistencia interna es el más utilizado por su simplicidad. Este método analiza la relación entre las diferentes partes de un mismo instrumento (Ledesma et al., 2002), y utiliza el coeficiente alfa de Cronbach (Cronbach, 1973) para valorar la consistencia del cuestionario completo o consistencia global. El grado de fiabilidad exigido, reflejado en el valor de este coeficiente, varía en función del tipo de estudio. En los estudios exploratorios, se exige que alcance un valor de 0.6, mientras que en el resto de los estudios se considera una escala fiable si el alfa de Cronbach es superior a 0.7. Este coeficiente se obtiene calculando las correlaciones entre los puntos, utilizando la siguiente fórmula matemática:

$r_{p p^{\prime}}=(K /(K-1))^{\star}\left(1-\left[\left(\sum X_{i i}\right) /\left(\sum X_{i i}+\sum X_{i j} \quad\right.\right.\right.$ donde $\left.\left.\left.i \neq j\right)\right]\right)$

Donde: $\mathrm{K}$ es el número de artículos del cuestionario, $\mathrm{X}_{\mathrm{ij}} \mathrm{y} \mathrm{X}_{\mathrm{ii}}$ son los elementos de la matriz de covarianza o la matriz de correlación. $\Sigma X_{i i}$ expresa la sumatoria de los elementos de la diagonal de la matriz y $\Sigma \mathrm{X}_{\mathrm{ii}+} \Sigma \mathrm{X}_{\mathrm{ij}}$ expresa la sumatoria de todos los elementos de la matriz.

Para el caso del instrumento de evaluación del módulo II, la fiabilidad obtenida es:

$r_{\mathrm{pp}}=(20 / 19) *(1-[20 /(20+2 * 41,77])=0,85$

Este valor proporciona seguridad respecto de que las acciones a tomar reflejarán realmente las necesidades de los alumnos. 


\section{CONCLUSIONES}

Del análisis y discusión de los resultados mostrados, se pueden obtener las siguientes conclusiones respecto de las potencialidades de la metodología MACCAD: 1) permite evaluar la calidad de las estrategias didáctico/pedagógicas propuestas en cursos bajo la modalidad a distancia, utilizando los indicadores media y dispersión; 2) establece el criterio o criterios que están más relacionados con la satisfacción general de los alumnos, mediante el análisis de correlación; 3) determina los criterios que requieren atención especial, esto es aquellos ya que siendo significativos para los alumnos reflejaron poco nivel de satisfacción; 4) identifica fortalezas y debilidades del curso; 5) permite evaluar la fiabilidad de los instrumentos de medición utilizados.

\section{REFERENCIAS}

Crosby, P.; Calidad para el Siglo XXI, pp 105, McGraw Hill, Bogotá, Colombia (1998).

Cronbach, L.; On estimates of test reliability. The Journal of Educational Psychology: 34 (4), 485494 (1973).

Garcia Aretio, L.: La Educación a Distancia. De la teoría a la práctica, 2a ed., 95-120, Ariel Educación, Barcelona, España (2002).

Hayes, B.; Measuring Customer Satisfaction ASQ Quality Pres, Mistwakee Wisconsin,USA (1992).

Herrera, M y otros tres autores; Diagnóstico de la calidad Educativa en Ciencias Básicas desde la perspectiva del alumno. $6^{\circ}$ Congreso de Informática del Nuevo Cuyo, San Juan, Argentina, 22 a 24 de Mayo 2007, http://www.unsj-cuim.edu.ar/portalzonda/congreso/ LpArea.asp (2007).

Juran, J.M. y F.M. Gryman; Análisis y planificación de la calidad, pp 55, McGraw Hill, México (1995).

Ledesma, R y otros 2 autores; Análisis de consistencia interna mediante Alfa de Cronbach: un programa basado en gráficos dinámicos (en línea), Noviembre de 2006, http://pepsic.bvspsi.org.br/pdf/psicousf/ v7n2a03.pdf (2002).

Litwin, E.(Compiladora); La Educación a Distancia. Temas para el debate en una nueva agenda educativa, 50-75, Amorrortu Editores S.A, Buenos Aires, Argentina (2000).

Rubio, M.J; Enfoques y Modelos de evaluación del e-learning (en línea), Relieve: 9(2), 2003, http://www.uv.es/RELIEVE/ v9n2/RELIEVEv9n2_1.htm (2003).

Spearman, C.; Correlation calculated from faulty data, British Journal of Psychology: 3 (2), 271295, (1930). 